\title{
Recurrent haemorrhagic ascites secondary to endometriosis: case report
}

\author{
Christian Alabi • Isaac Evbuomwan • \\ Stephen Attwood • Jonathan Brady
}

Received: 28 January 2007 / Accepted: 15 March 2007 / Published online: 17 April 2007

(C) Springer-Verlag 2007

\begin{abstract}
We present a case of a young black African woman who presented with recurrent haemorrhagic ascites secondary to endometriosis. This is a rare entity which can be easily confused with bowel or ovarian malignancy and the few reported cases have been managed by laparotomy unlike our case which was managed purely with laparoscopic surgery.
\end{abstract}

Keywords Massive haemorrhagic ascites · Endometriosis . Ascites and endometriosis $\cdot$ Massive ascites

\section{Introduction}

Haemorrhagic ascites associated with endometriosis is uncommon, and reported cases in the European literature are scant. It is a diagnosis usually made after exhaustive exclusion and often after a surgical exploration of the abdomen has been undertaken. Most of the reported cases have involved nulliparous black women. This entity simulates gynaecological malignancy and often involves

\author{
C. Alabi \\ Hope Hospital, \\ Salford, UK \\ I. Evbuomwan \\ Queen Elizabeth Hospital, \\ Gateshead, UK \\ S. Attwood $\cdot$ J. Brady \\ North Tyneside General Hospital, \\ North Tyneside, UK \\ C. Alabi $(\bowtie)$ \\ Stott Lane, \\ Salford Manchester, Lancashire M6 8HD, UK \\ e-mail: christianalabi@aol.com
}

multiple disciplines. We describe such a case of a black woman who presented with recurrent haemorrhagic ascites and who had just commenced a cycle of in vitro fertilisation (IVF) treatment. Consistent with other reported cases in the literature, this proved to be a diagnostic dilemma to us and colleagues in other disciplines. The case also highlights the usefulness of advanced laparoscopic surgery in obviating the need for exploratory laparotomy as used in other cases.

\section{Case report}

Mrs B.O., a 30-year-old married woman of black African origin, was diagnosed as having vaginal endometriosis following biopsy of a vaginal nodule 4 years prior to presentation.

Diagnostic laparoscopy later performed in view of a history of subfertility, deep dyspareunia, cyclical rectal pain and severe backache demonstrated endometriotic deposits in the pouch of Douglas and right ovary, the left tube and ovary were fixed and densely adherent to the pelvic side wall and the left adnexum obscured by omental adhesions.

Superficial laser vaporisation of posterior vaginal wall endometriosis was performed and she was commenced on a 6-month course of treatment with a gonadotrophin-releasing hormone analogue (GnRHa), goserelin (Zoladex, AstraZeneca). Her symptoms improved on Zoladex but returned following cessation of treatment. She was subsequently referred for IVF treatment (3-year history of secondary subfertility and severe endometriosis).

Within 3 days of commencing pituitary down-regulation treatment for IVF using GnRH agonist, she was admitted into hospital. She presented with abdominal distension and pain. Investigations undertaken included ultrasound (USS) of the pelvis and abdomen and a computed tomographic 
(CT) scan which demonstrated gross ascites, some bilateral basal pleural thickening, no intra-peritoneal or pelvic lymphadenopathy, minimally enlarged ovaries, normal liver, spleen, pancreas, gall bladder and kidneys. There were no peritoneal plaques. Other investigations included Ca 125 [56 (normal: <35)], C-reactive protein [388 (normal: <5)] and haemoglobin $8.5 \mathrm{~g} / \mathrm{dl}$. Haemorrhagic fluid from a diagnostic paracentesis proved negative for malignant cells, but contained numerous haemosiderinladen cells.

Her IVF treatment was abandoned at this stage. She returned home after a week, but was readmitted into hospital because of persistent abdominal distension and pain. Her subsequent management was multi-disciplinary and included a gynaecologist, general surgeon and a physician. Emergency diagnostic laparoscopy was performed and 51 of haemorrhagic fluid were drained. There were also endometriotic lesions on the right ovary, with the left adnexum buried in a mass of small and large bowel. As she was not bowel prepared, exploratory surgery was not carried out.

At exploratory laparoscopy (Fig. 1) undertaken 1 week later, the sigmoid colon, left ovary and fallopian tube were successfully mobilised using Harmonic Scalpel. She made a good recovery and was discharged home 2 days later. Culture of ascitic fluid drained grew Mycoplasma hominis and she was treated with doxycycline for 2 weeks. There was no evidence of tuberculosis (TB).

She was re-admitted 2 months later with a recurrence of ascites, confirmed on USS. She had paracentesis and 2,500 ml of haemorrhagic fluid were drained. She represented a month later with the same symptom and a more extensive exploratory laparoscopy was therefore arranged in order to confirm no pathology was missed the last time as she was still not better clinically.

Then 1,500 $\mathrm{ml}$ of haemorrhagic fluid were drained and there were endometriotic deposits throughout the pelvis, with the sigmoid colon being adherent to the left fallopian tube and left ovary. The right ovary was adherent to the pelvic side wall. Separate endometriotic implants were found on the caecum, anterior peritoneal wall and a nodule in the lesser sac. There were extensive adhesions between the liver and the diaphragm. The lesser sac, pancreas and the small bowel in its entire length otherwise appeared normal.

Multiple biopsies obtained from the pelvis, liver capsule, and peritoneum confirmed endometriosis, while the lesser sac nodule was reported as containing lots of haemosiderinladen lymphocytes as did cytology of the ascitic fluid with no neoplastic cells.

The sigmoid colon was successfully mobilised along with the left fallopian tube and left and right ovary which were only partially mobilised. She made a good recovery and was discharged home 2 days later. She later conceived spontaneously whilst contemplating another attempt at having IVF treatment and she has since had a live term baby.

\section{Discussion}

Haemorrhagic ascites complicating endometriosis was first described by Brews in 1954 [1]. It remains an uncommon phenomenon, with about 41 cases having been reported since its first description [2]. Most of the reported cases are from the American literature, with a few in the Asian
Fig. 1 Extensive exploratory laparoscopy. a Pool of haemorrhagic ascitic fluid partially covering loops of bowel. b Endometriotic implant on large bowel surface. c Dense adhesions between liver and diaphragm. d Liver with endometriotic implants and pool of ascitic fluid in background
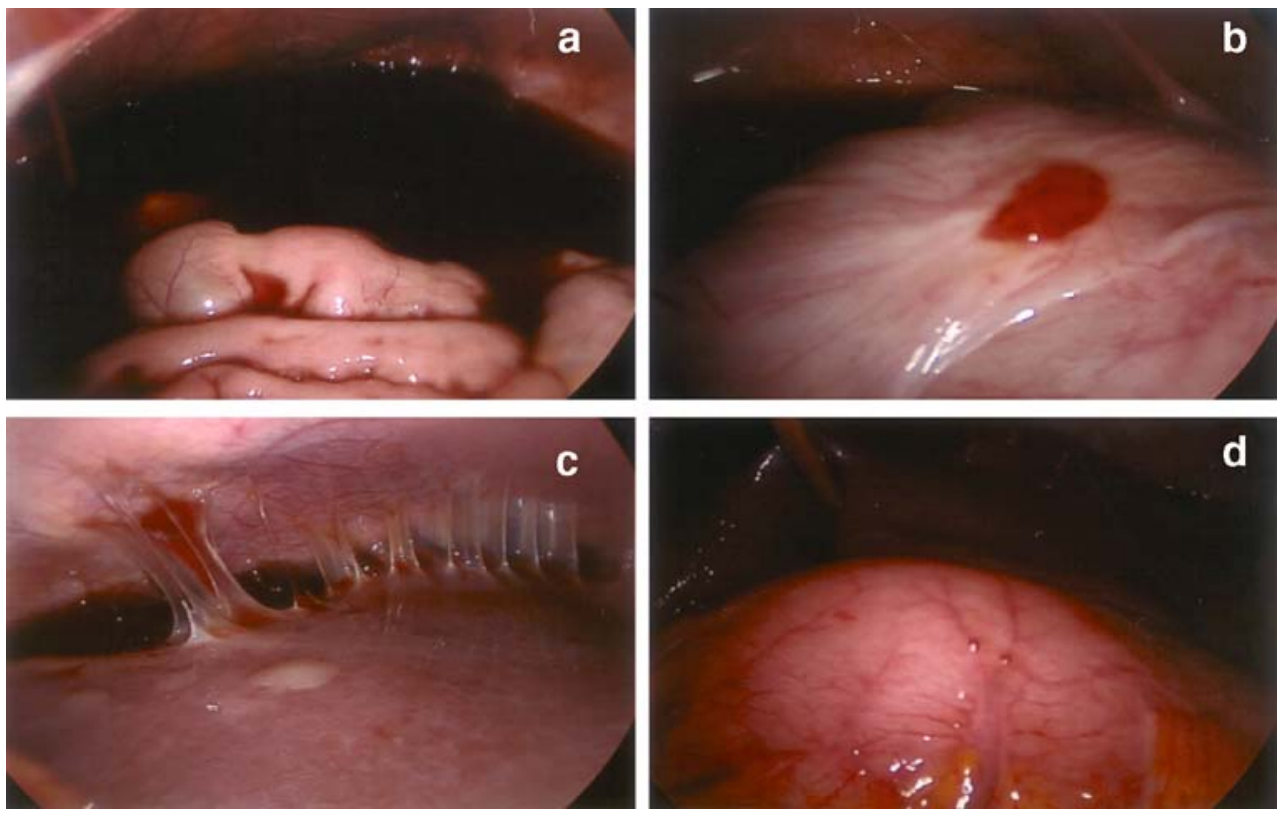
literature. To our knowledge, there has been no reported case in the UK.

This entity seems to have a predilection for black, nulliparous women, with most of the women being in their reproductive age [3, 4]. It often poses a diagnostic dilemma when it presents, the diagnosis only being established after exhaustive exclusion of other important conditions such as malignancy (especially bowel and ovarian neoplasia). The aetiology of the haemorrhagic ascites in endometriosis is still unknown. There has been one report in the literature which has attempted to link this aetiology to ovulation induction [5].

Indeed, one is tempted to also suggest such a link in our own case, since she had only just commenced treatment for IVF. However, such a link would be very unlikely, given that she only had 3 days of GnRHa, and the onset of her symptoms did pre-date her commencement of IVF treatment by about 2 months. A high index of suspicion is required for prompt diagnosis and further treatment. Laparoscopy is the gold standard to confirm endometriosis and symptomatic relief of gross abdominal distension by drainage of significant ascites can be achieved (as in our case) at the same time. Although ultrasound-guided paracentesis can also be used for symptomatic relief of massive ascites laparoscopy per se is not required. Once the diagnosis is confirmed, the main difficulty is deciding which treatment modality is better suited. The obvious choice as in this case would have been GnRHa, as in similarly young nulliparous women, and in fact a complete remission was described in one of the cases [6].

However, using this treatment modality in this case would have further delayed her infertility treatment and we speculate our patient may have inadvertently been stimulated by the flare-up effect that initially occurs during pituitary down-regulation with GnRHa.
In a woman who has completed her family, options can include laparotomy and extensive excision of endometriosis or in units where the expertise is available, laparoscopic surgery may be more attractive or suitable.

\section{Conclusion}

Haemorrhagic ascites complicating endometriosis remains a rare but very important entity because of the diagnostic dilemma it poses and its management implications especially in women in the reproductive age who wish to preserve their fertility.

It is clear that this entity does exist, and although most clinicians may never encounter a single case, it nevertheless should be borne in mind as a differential when evaluating a young patient, who presents with ascites and a history suggestive of endometriosis.

\section{References}

1. Brews A (1954) Endometriosis including endometriosis of the diaphragm in Meigs syndrome. Proc R Soc Med 10:309-312

2. Ekoukou D, Guiherne R, Desligneres S, Rotten D (2005) Endometriosis with massive hemorrhagic ascites: a case report and review of the literature (in French). J Gynecol Obstet Biol Reprod (Paris) 34(4):351-359

3. Jenks JE, Artman LE, Hoskin WJ, Mireradi AK (1984) Endometriosis with ascites. Obstet Gynecol 63(3 Suppl):75S-77S

4. Cheong ES, Lim DTH (2003) Massive ascites - an uncommon presentation of endometriosis. Singapore Med J 44(2):98-100

5. Feigin RD, Glikson M, Gur H, Galun E, Younis JF, Beyth Y (1988) Induction of ovulation causing recurrent bloody ascites in a woman with endometriosis. Am J Obstet Gynecol 159(5):1161-1162

6. Dias CC, Andrade JM, Ferriani RA, Villanova MG, Meirelles RS (2000) Hemorrhagic ascites associated with endometriosis. A case report. J Reprod Med 45(8):688-690 